\title{
COMPLETION PNEUMONECTOMY: CURRENT INDICATIONS, COMPLICATIONS, AND RESULTS
}

Toshio Fujimoto, MD

George Zaboura, MD

Sylvia Fechner, MD

Ludger Hillejan, MD

Thomas Schröder, MD

Alessandro Marra, MD

Thomas Krbek, MD

Marc Hinterthaner, MD

Dieter Greschuchna, MD

Georgios Stamatis, MD
Objective: Completion pneumonectomy is reported to be associated with high morbidity and mortality, especially when done in patients with benign disease. We review our 9 years of experience with this operation to evaluate the postoperative outcome and long-term results of various indications.

Methods: Between January 1990 and December 1998, 66 consecutive patients underwent completion pneumonectomy (6.8\% of all pneumonectomies), and their cases were retrospectively reviewed. The indication was benign disease in 17 patients and malignant disease in 49 patients. In patients with malignant indications there were 14 local recurrences, 4 second primary tumors, 5 metastatic diseases, and 26 indications because of incomplete initial resection.

Results: There were no intraoperative deaths, and the postoperative mortality rate was $7.6 \%$. Complications were encountered in $32(53 \%)$ patients, without any significant difference between benign indication $(71 \%)$ and malignant indication (47\%; $P=.0923)$. Bronchopleural fistula was encountered in $5(7.6 \%)$ patients, and empyema was encountered in $7(11 \%)$ patients. The actuarial 5-year survival was $57 \%$ for all patients, $65 \%$ for those with benign indications, and 54\% for those with malignant indications (60\% for local recurrence, $50 \%$ for second primary tumor, and $56 \%$ for incomplete resection), without any difference between benign and malignant indications $(P=.9478)$.

Conclusions: Completion pneumonectomy can be performed with acceptable mortality and morbidity, even in patients with benign disease. Patients with preoperative infection can be managed with bronchial stump covering and adequate postoperative drainage. Although complications are common, they can successfully be managed with a proper understanding of them. ( $\mathrm{J}$ Thorac Cardiovasc Surg 2001;121:484-90)

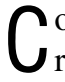
ompletion pneumonectomy refers to a procedure to remove what is left of a lung partially resected during a previous operation. Although it is technically demanding and associated with significant morbidity and mortality, ${ }^{1}$ indications for malignant disease have increased with regard to the increasing frequency of lung cancer, the widespread use of limited resection for early lung cancer, and the increasing demands for pulmonary resection for pulmonary metastases. Since the first detailed report by McGovern and colleagues, ${ }^{2}$ many reports have been published to examine the risk and outcome of this technique. In most series the pro-

From the Department of Thoracic Surgery and Endoscopy, Ruhrlandklinik, Essen-Heidhausen, Germany.

Copyright (C) 2001 by The American Association for Thoracic Surgery

0022-5223/2001 $\$ 35.00+0 \quad \mathbf{1 2 / 1 / 1 1 2 4 7 1}$

doi:10.1067/mtc.2001.112471 cedure could be performed with acceptable mortality, although it had a higher morbidity, especially when done in patients with benign disease. ${ }^{3-7}$ We review our 9 years of experience with this operation to evaluate the postoperative outcomes and long-term results of various indications.

\section{Patients and methods}

Between January 1990 and December 1998, 66 consecutive patients underwent completion pneumonectomy for a variety of pulmonary diseases in the Department of Thoracic Surgery and Endoscopy of Ruhrlandklinik in EssenHeidhausen. This represented $6.8 \%$ of all pneumonectomies (967 cases) done during the same period. There were 44 male and 22 female patients, with an average age of 57 years (range, 37-72 years). Right completion pneumonectomy was done in 33 patients, and left completion pneumonectomy was done in 33 patients.

First operation. Indications for initial surgical procedure were benign diseases in 10 patients (bronchiectasis, 5; 
tuberculosis, 3; bronchopulmonary aspergillosis, 1; and pulmonary dermoid cyst, 1) and malignant diseases in 56 patients (primary lung cancer, 46; metastatic lung cancer, 7; carcinoid tumor, 3). TNM classification of the patients with primary lung cancer was T1 N0 in 4 patients, T2 N0 in 14 patients, $\mathrm{T} 1 \mathrm{~N} 1$ in 2 patients, $\mathrm{T} 2 \mathrm{~N} 1$ in 17 patients, T2 $\mathrm{N} 2$ in 1 patient, T3 N0 in 1 patient, T3 N1 in 3 patients, T2 N3 in 3 patients, and T4 NO in 1 patient. The initial surgical procedure was a right upper lobectomy in 17 patients, a right lower lobectomy in 5 patients, a right upper sleeve lobectomy in 3 patients, a right upper bilobectomy in 2 patients, a right lower bilobectomy in 3 patients, a right partial resection in 3 patients, a left upper lobectomy in 14 patients, a left lower lobectomy in 14 patients, a left segmental resection in 3 patients, and a left partial resection in 2 patients. Five $(7.6 \%)$ patients underwent postoperative radiation therapy up to $60 \mathrm{~Gy}$.

Completion pneumonectomy. The indication for completion pneumonectomy was benign disease in 17 patients (bronchiectasis, 7; resistant pneumonia, 3; bronchopleural fistula [BPF], 2; lung abscess, 2; lung hemorrhage, 2; and tuberculosis, 1) and malignant disease in 49 patients. In the group with malignant disease, including carcinoid tumor, there were 14 local recurrences, 4 second primary tumors, 5 metastatic diseases, and 26 indications because of incomplete initial resection revealed by means of postoperative pathologic findings (R1). The relationship between the first and second operation is shown in Table I.

Local recurrence. Local recurrence is defined as a second lung malignancy with the same histologic features and the same anatomic site, occurring within 2 years of the first operation. ${ }^{5}$ Stump recurrence is regarded as a local recurrence regardless of the time interval. Among the patients, 9 had adenocarcinomas, 1 squamous cell carcinoma, 1 adenosquamous cell carcinoma, 1 large cell carcinoma, and 2 carcinoid tumors. TNM classifications at initial operation were T1 N0 M0 in 2 patients, T2 N0 M0 in 8 patients, and T1 N1 M0, T2 $\mathrm{N} 1 \mathrm{M} 0, \mathrm{~T} 2 \mathrm{~N} 2 \mathrm{M} 0$, and T3 N1 M0 in 1 patient each. The mean interval between the first and second operation was 34 months (range, 3-74 months).

Second primary tumor. Second primary tumor is defined as a second malignancy with a different histologic type, different anatomic site, and occurrence after more than 2 years from the first malignancy. ${ }^{8}$ Histologic and TNM classification of the included patients were as follows (initial histology and stage): adenocarcinoma, T2 N0 (adenocarcinoma, T2 N0 14 years before); adenocarcinoma, T2 N0 (squamous cell carcinoma, T1 N0 4 years before); and squamous cell carcinoma, T3 N1 (squamous cell carcinoma, T2 N1 9 years before). A case of primary lung cancer (adenocarcinoma, T2 N1 M1) after pulmonary resection of metastatic testicular carcinoma 4 years before was included for a convenience.

Metastatic lung tumor. Primary tumor included pharyngeal cancer in 2 patients and colon cancer, rectal cancer, and malignant schwannoma in 1 patient each. The mean interval between the first and second operations was 16 months (range, 4-25 months). All patients were free from metastasis of the hilar and mediastinal lymph nodes, except for one.
Incomplete initial resection. Because of the incomplete initial resection revealed by postoperative pathology (R1), some patients underwent completion pneumonectomy, although this indication has recently diminished because of use of intraoperative frozen sections. The reason for incomplete initial resection was due to positive bronchial margin in 15 patients and positive N1 lymph node in 11 patients, whose extracapsular infiltration could not be found intraoperatively but was proven pathologically. These included 11 adenocarcinomas, 12 squamous cell carcinomas, 1 adenosquamous cell carcinoma, 1 large cell carcinoma, and 1 adenoid cystic carcinoma. TNM classification of the patients was T1 N0 in 2 patients, T2 N0 in 5 patients, T1 N1 in 1 patient, T2 N1 in 17 patients, and T3 N1 in 1 patient. No tumor component was found in the specimen of reoperation in 18 of 26 patients. Histopathologically, the tumor reached immediately to the resection margin of the first operation but not beyond it. The mean interval between the first and second operations was 28 days (range, 8-89 days).

In the group with benign disease, there were 4 indications because of early complication (within 30 days after the first operation) of the initial procedure, which included 3 patients with uncontrollable pneumonia that required an urgent resection to avoid septicemia and 1 patient with hemorrhagic necrosis of the lung. The time intervals between the first and second operations were 2, 8, 9, and 11 days. For all benign indications, the mean interval between the first and second operation was 133 months (range, 0-519 months).

Operative technique. Inclusion for completion pneumonectomy was restricted to patients with expected postoperative forced expiratory volume in 1 second of more than 1.0 $\mathrm{L}$, as calculated by perfusion scintigraphy, and endurable cardiac function. In case of a second primary malignant tumor suspected preoperatively and in case of local recurrence occurring more than 2 years after initial procedure, repeated mediastinoscopy was performed as a rule to exclude $\mathrm{N} 2$ and N3 disease.

We chose anterolateral thoracotomy as a rule, and the same incision line as the initial operation was used for completion pneumonectomy. The pericardium was open to dissect hilar vessels when hilar adhesion was hard enough to dissect extrapericardially. As a result, intrapericardial vessel ligation was done in $41(62 \%)$ patients. The bronchial stump was manually sutured and covered with either intercostal muscle or mediastinal fat in all patients who had received radiation therapy after the initial operation ( 5 patients). Additional surgical procedures were necessary in $7(11 \%)$ patients: chest wall resection in 3 patients; carinal resection in 2 patients; pleuropneumonectomy in 1 patient; and pericardial resection with insertion of prosthesis in 1 patient. The mean intensive care unit stay was 4.4 days (range, 1-15 days). Mechanical ventilation for more than 1 day was needed in 18 (27\%) patients (mean, 2.2 days; range, 1-10 days).

Statistical analysis. All intrahospital records of patients were reviewed for clinical data, operative records were reviewed for intraoperative problems, and intensive care unit charts were reviewed for early postoperative complications. The unpaired Student $t$ test and the $\chi^{2}$ test were used for 
Table I. Relationship between indications of the first and second operation

\begin{tabular}{llll} 
& & \multicolumn{1}{c}{ Completion pneumonectomy } \\
\cline { 3 - 4 } First operation & No. & \multicolumn{1}{c}{ Indication } & No. \\
\hline Primary lung cancer & 46 & Incomplete resection & 26 \\
& & Local recurrence & 3 \\
& & Second primary lung cancer & 3 \\
& & Pneumonia & 1 \\
Metastatic lung tumor & & BPF & 1 \\
& & Lung abscess & 5 \\
Bronchiectasis & 7 & Metastatic lung tumor & 1 \\
Carcinoid tumor & & Second primary lung cancer & 1 \\
& & Lung abscess & 5 \\
Tuberculosis & 5 & Bronchiectasis & 2 \\
& 3 & Local recurrence & 1 \\
Bronchopulmonary aspergillosis & & Hemorrhagic necrosis & 1 \\
Other benign tumor & 3 & Tuberculosis & 1 \\
Total & & Bronchiectasis & 1 \\
\hline
\end{tabular}

Table II. Details of postoperative complications

\begin{tabular}{lrc}
\hline Type of complication & No. & $\%$ \\
\hline Cardiac arrhythmia & 14 & 21 \\
Empyema & 7 & 11 \\
Postoperative bleeding & 5 & 7.6 \\
BPF & 5 & 7.6 \\
Pneumonia & 3 & 4.5 \\
Pulmonary edema & 3 & 4.5 \\
Sputum retention & 2 & 3.0 \\
Chylothorax & 1 & 1.5 \\
Pneumothorax & 1 & 1.5 \\
Recurrent nerve paralysis & 1 & 1.5 \\
Total (patient) & 35 & $53 \%$ \\
\hline
\end{tabular}

group comparison when appropriate. Survival was estimated from the time of completion pneumonectomy with a KaplanMeier model. The comparisons of survival between subgroups were made with the log-rank test.

\section{Results}

There were no intraoperative deaths and 5 (4 in patients with benign indications and 1 in a patient with a malignant indication) early postoperative deaths within 30 days after the operation (mortality rate, $7.6 \%)$. In 3 of these cases, completion pneumonectomy was done because of early complication of the initial procedure. The cause of the deaths was multisystem failure caused by contralateral pneumonia in 3 patients, pulmonary embolism in 1 patient, and cardio- genic shock caused by postoperative bleeding in 1 patient.

Complications after completion pneumonectomy were encountered in 35 (53\%) patients (Table II). Complications occurred in $12(71 \%)$ patients of the group with benign indications and $23(47 \%)$ patients of the group with malignant indications, although this difference was not significant $(P=.0923)$.

BPF was encountered in $5(7.6 \%)$ patients, and empyema was encountered in $7(11 \%)$ patients. The mean intervals between completion pneumonectomy and BPF occurrence was 36 days (range, 6 days- 4 months). One of 5 (20\%) patients who received radiation therapy before completion pneumonectomy had a BPF, a higher rate than the $6.6 \%$ of patients who had not. In 3 of 5 patients with BPFs, thoracostomas and subsequent thoracoplasties were performed because nonsurgical procedures were ineffective. There were no statistically significant differences in the occurrence rate of empyema between the group with benign indications (3/17 [18\%] patients) and the group with malignant indications (4/45 [8.9\%] patients; $P=.2739)$. The bacteria detected in the empyema cavity were Streptococcus species in 2 patients, Pseudomonas species in 2 patients, and Acinetobacter and Candida species in 1 patient each. In the remaining patient no bacterium was detected.

Cardiac rhythm dysfunctions were encountered in 14 (21\%) patients, all of which were classified as supraventricular arrhythmia. The initial onset of 


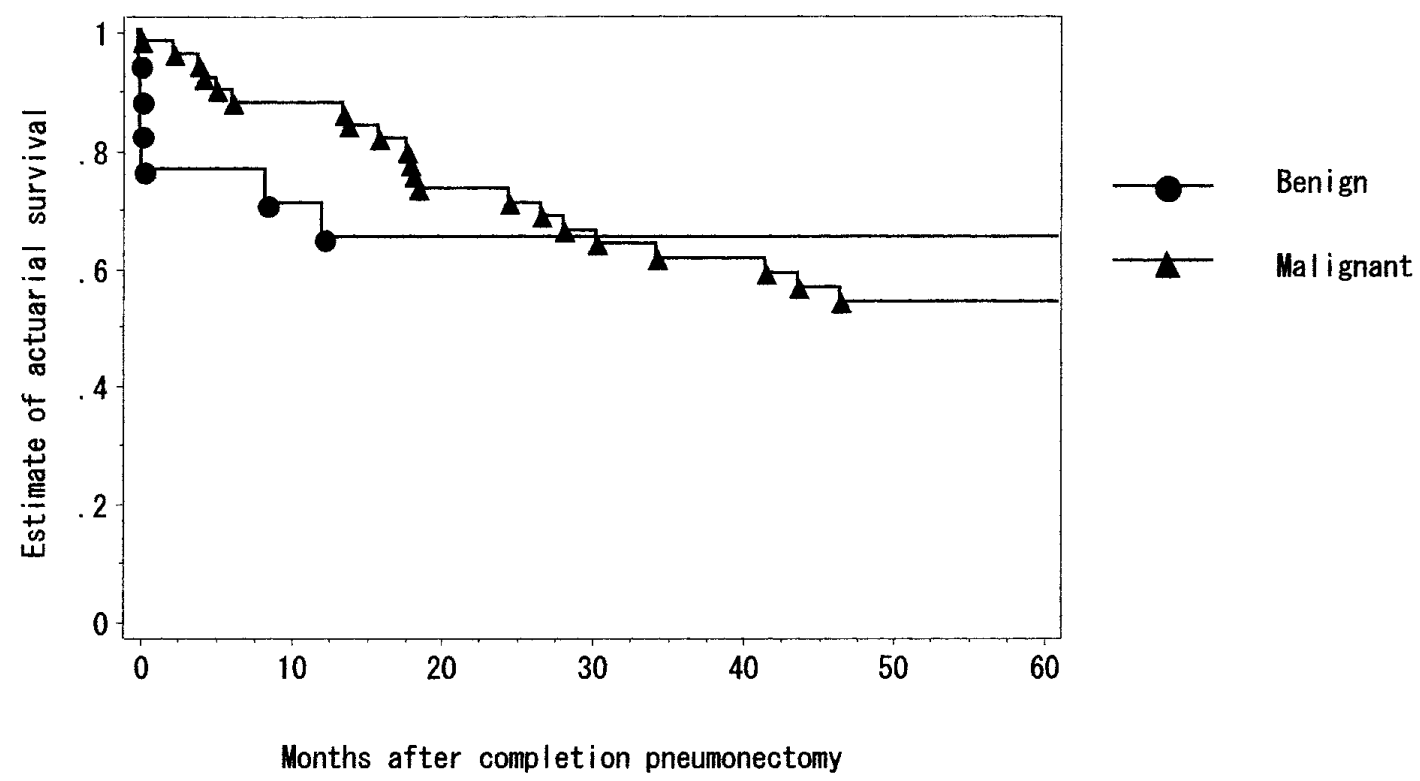

Fig 1. Analysis of survival related to indications for completion pneumonectomy.

arrhythmia occurred between postoperative days 1 and 14 (median, day 2). These were all successfully treated with digoxin and calcium blockers.

The mean operative time was 170 minutes (range, 67345 minutes), and the mean blood loss during the procedure was $805 \mathrm{~mL}$ (range, 100-3400 mL). The mean blood loss in the group with benign indications was 973 $\mathrm{mL}$, and that in the group with malignant indications was $752 \mathrm{~mL}$, although the difference was not statistically significant $(P=.2219)$. Major blood vessel injury occurred in $5(7.6 \%)$ patients. The mean hospital stay was 23 days (range, 14-184 days).

Follow-up was completed for all patients, and the mean follow-up period of patients who survived more than 30 days postoperatively was 4.5 years (range, 2115 months). Actuarial survival was calculated from the time of completion pneumonectomy (Figs 1 and 2). The actuarial 5-year survival was $57 \%$ for all 66 patients, $65 \%$ for patients with benign disease and $54 \%$ for patients with malignant disease, without any difference between patients with benign disease and malignant disease $(P=.9478)$. We also analyzed survival with regard to the indications in patients with malignant disease. The actuarial 5-year survival was $60 \%$ for the local recurrence group, $50 \%$ for the second primary tumor group, and 56\% for the incomplete initial resection group. The survival of the patient in the metastatic lung tumor group has not reached 5 years. There were no significant differences between groups $(P=.4288)$.

\section{Discussion}

Completion pneumonectomy is a challenging procedure that poses a great stress on surgeons not only intraoperatively but also preoperatively and postoperatively. The surgeon cannot be too careful in deciding whether the operation is indeed indicated, whether the gain in survival and quality of life outweighs the risks involved, and whether other therapeutic options are available, as stated by Deslauriers. ${ }^{9}$

Snijder and colleagues ${ }^{10}$ classified residual tumor as carcinoma in situ (CIS), mucosa residual disease (MRD), peribronchial residual disease (PRD), or lymphatic infiltration. They concluded that patients with invasive tumors (MRD+PRD) have a high incidence of local recurrence and that such tumors have an adverse effect on survival. In their series the 5-year survival in the complete resection group was $54 \%$, whereas our result in the same group was $56 \%$. According to this classification, our patients had no CIS, 5 had MRD, 10 had PRD, and 11 had lymphatic infiltration (positive N1 lymph node that could not be extirpated with a capsule). Our policy is that patients with either CIS or invasive tumor, including lymphatic infiltration at the resection margin, should undergo reoperation, especially for early-stage lung cancer, whenever their conditions are tolerable. As to lymphatic infiltration, if the lymph node is extirpated with a capsule and R0 resection can be achieved, we will not go so far as pneumonectomy because no superiority of pneumonectomy to lobectomy has been observed for N1 disease. ${ }^{11,12}$ 


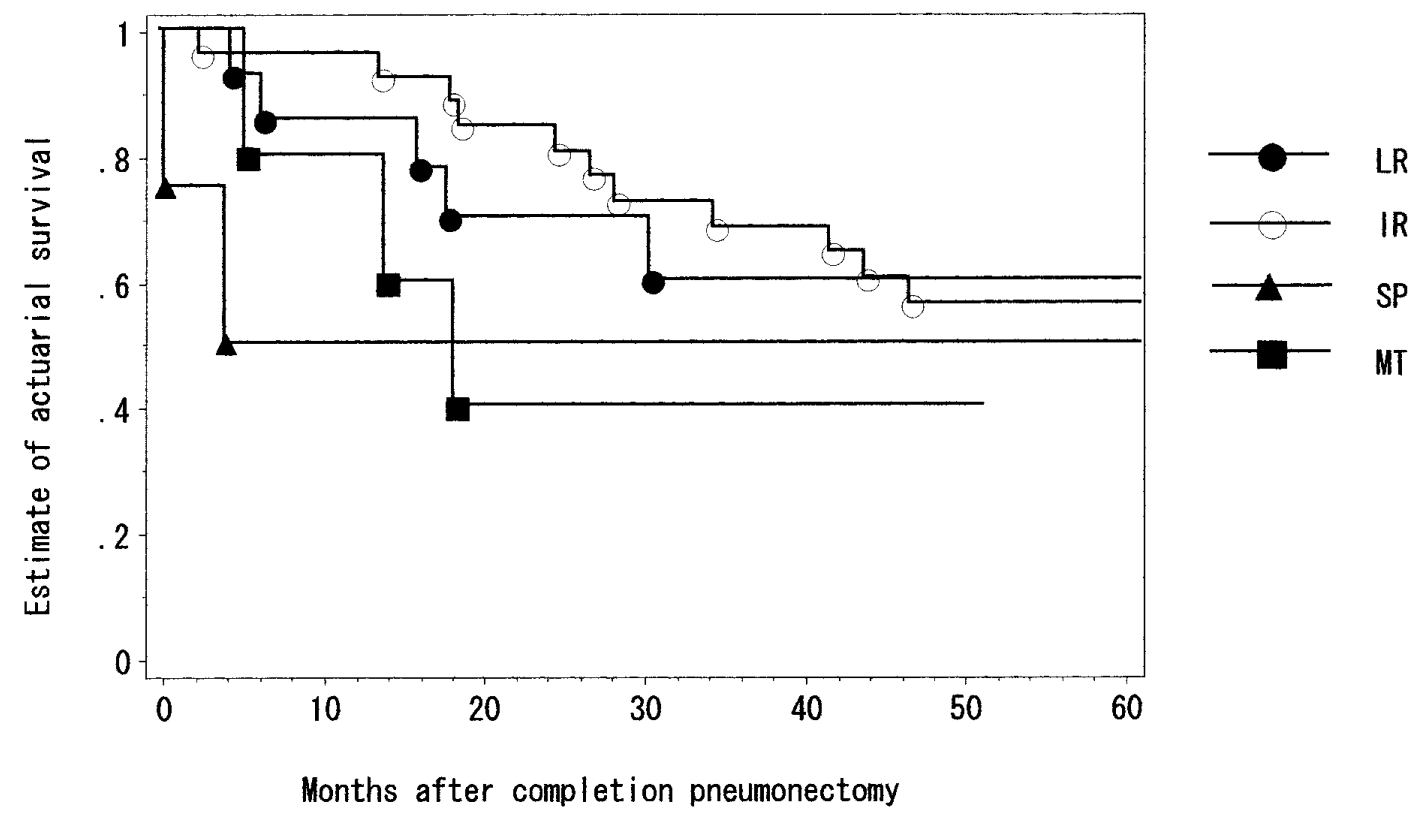

Fig 2. Analysis of survival in the group with malignant indications. $L R$, Local recurrence; $I R$, incomplete resection; $S P$, second primary lung cancer; $M T$, metastatic lung tumor.

In our results the patient group that underwent completion pneumonectomy for an early complication of initial procedure showed significantly higher mortality (3/4 [75\%] patients). As suggested by Muysoms and colleagues, ${ }^{3}$ who reported a mortality rate of $37.5 \%$ for the same indication, completion pneumonectomy should be performed for this group only if there is no alternative method of therapy and only after sincere explanations to patients and families of this extremely high risk.

Resection of pulmonary metastases by means of pneumonectomy remains under discussion. Our data are perhaps too minimal to induce any conclusions, but the presence of survivors over 4 years suggests that the procedure should not be considered an absolute contraindication if the patients are selected by means of strict criteria. ${ }^{13}$ Our criteria for eligibility included the following: motivated patients under 70 years old; stabilized primary tumor; absence of mediastinal lymphadenomegaly detected by means of computed tomography; no extrathoracic metastases at the time of thoracotomy; acceptable cardiorespiratory reserve; and no alternative treatment other than operation.

Completion pneumonectomy for bronchiectasis is said to be a relatively high-risk procedure, and its mortality can be as high as $43 \% .{ }^{14}$ However, the mortality rate in our series was $0 \%$, and the severe complication (BPF) occurred in $14 \%$ of the patients. These figures were acceptable, taking into consideration that we included the emergency case of uncontrollable bleeding. Complete resection of the affected segments is most important for the avoidance of a second operation. All of our patients had undergone complete resection at the initial operation, but new lesions, which could not be detected by bronchography before, appeared over 20 years.

Repeated mediastinoscopy may be effective in restricting cases of second primary lung cancer and metastatic lung tumor to inclusion to exclude N2 and $\mathrm{N} 3$ disease, which is afforded little benefit with this risky operation.

Radiation therapy precedes the hilar adhesion and may obliterate the intrapericardial plane, which makes the operation more difficult. We should consider the possibility of having to reoperate on a patient who has had complete resection of lung cancer before postoperative radiation therapy because the effectiveness of it is not clear. ${ }^{4}$ Deslauriers 9 reported the "bronchus first" technique in case of severe hilar adhesion. This technique may especially be effective in completion pneumonectomy after radiation therapy, but we did not need to use it for the cases reported.

Complications after completion pneumonectomy are common. The complication rate of our data was $53 \%$, a little higher than those reported previously (18\%$46 \%) .^{2-7}$ This is probably because of our inclusion of nonsevere arrhythmia, as detected by Holter electrocardiography recording in our intensive care unit. We 
observed postoperative supraventricular arrhythmia in $21 \%$ of the patients. Some authors stated that the extent of pulmonary resection was correlated with the development of this type of arrhythmia, ${ }^{15}$ whereas others observed the strong relationship of arrhythmia only with pneumonectomy (incidence, 46.1\%). ${ }^{16}$ In any case, we recommend the prophylactic treatment of the patient who is planning on undergoing completion pneumonectomy.

BPFs occurred in 5 (7.6\%) patients, 4 of which were on the right side, 1 of which occurred with preoperative radiation, and 1 of which occurred on the left side with preoperative severe infection. This rate is similar to those of previous reports $(0 \%-13 \%){ }^{2-7}$ In our series there was no significant difference of the BPF occurrence rate between the group with benign disease and that with malignant disease, as opposed to the results of previous reports. We have routinely covered the bronchial stump with a flap of intercostal muscle or mediastinal fat in case of right side and preoperative radiotherapy. ${ }^{17}$ In addition, covering of the bronchial stump of the patients with preoperative infection is recommended.

As to empyema, we have not observed any significant difference in the occurrence rate between patients with preoperative infection and patients without it (13\% vs $10 \%, P=.6964)$. Patients with preoperative infection can be successfully managed with preoperative prophylactic antibiotic therapy, intensive respiratory physiotherapy, intraoperative stump-covering technique, and postoperative washing of the thoracic cavity through drainage, which should be kept in place until the thoracic cavity proves to be sterile postoperatively. Al-Kattan and Goldstraw ${ }^{5}$ advised an additional surgical procedure, such as a limited thoracoplasty with a use of an omental or muscle flap, for cases involving infection.

Contralateral pneumothorax may potentially be a life-threatening complication. The possibility of such an occurrence would be higher because mechanical ventilation is frequently and for a longer time used for respiratory failure after pneumonectomy. We must carefully check contralateral pneumothorax by means of daily roentgenography in addition to contralateral pneumonia.

Chylothorax develops in $0.2 \%$ to $0.5 \%$ of cardiovascular and thoracic operations, ${ }^{18}$ and its occurrence after pneumonectomy is rare. We must, however, pay attention to the excessive and rapid filling of the postpneumonectomy space in the early postoperative period, which may be due to bleeding, infection, and chylothorax. ${ }^{19}$ Chylothorax should be treated early by means of a surgical invention because it can easily deteriorate the hemodynamic and respiratory state of a patient after pneumonectomy. Our patients with this complication showed rapid filling of the thoracic cavity and needed a surgical procedure to control the chylous leak at postoperative day 5 .

Completion pneumonectomy should be done by skilled thoracic surgeons. Major technical difficulties in operations exist in the dissection of the pleural adhesion, which sometimes needs to be done through the extrapleural plane. Major blood vessel injury occurred in $5(7.6 \%)$ patients, and the mean bleeding volume was $805 \mathrm{~mL}$. These values are lower than those in most previous reports $(3.8 \%-16.2 \%$ and $950-$ $2019 \mathrm{~mL}$ each), ${ }^{2-7}$ which perhaps is a result of our inclusion of the incomplete resection group, which had a smaller interval between the first and second operation and consequently had a significantly lower bleeding volume than the rest of the groups $(563 \mathrm{~mL}$ vs 978 $\mathrm{mL}, P=.0061)$.

In contrast to a previous report, ${ }^{2}$ we did not observe any difference in postoperative mortality between patients with benign disease and malignant disease. The mortality rate in our series was $7.6 \%$, which is comparable with the figures shown for standard pneumonectomy in the literature. ${ }^{20}$

In our study we showed an overall 5 -year survival of $54 \%$ among patients with malignant disease. This high survival compared with the rate of $23 \%$ to $44.5 \%$ reported previously ${ }^{5-12}$ was probably related to the inclusion of patients in the incomplete initial resection group, most of whom had early stage lung cancer. Nonetheless, these favorable long-term results justify the procedure for the treatment of disease with severe prognosis.

Received for publication June 6, 2000; revisions requested Sept 8, 2000; revisions received Sept 21, 2000; accepted for publication Oct 24, 2000.

Address for reprints: Toshio Fujimoto, MD, Department of Thoracic Surgery and Endoscopy, Ruhrlandklinik, Tüschener Weg 40, 45239 Essen, Germany (E-mail: NanakoFjmt@aol.com).

\section{REFERENCES}

1. Tronc F, Grégoire J, Rouleau J, Deslauriers J. Techniques of pneumonectomy: completion pneumonectomy. Chest Surg Clin N Am 1999;9:393-405.

2. McGovern EM, Trastek VF, Pairolero PC, Payne WS. Completion pneumonectomy: indications, complications and results. Ann Thorac Surg 1988;46:141-6.

3. Muysoms FE, de la Riviére AB, Defauw JJ, Dossche KM, Knaepen PJ, van Swieten HA, et al. Completion pneumonectomy: analysis of operative mortality and survival. Ann Thorac Surg 1988;66:1165-9. 
4. Grégoire J, Deslauriers J, Guojin L, Rouleau J. Indications, risks, and results of completion pneumonectomy. J Thorac Cardiovasc Surg 1993;105:918-24.

5. Al-Kattan K, Goldstraw P. Completion pneumonectomy: indications and outcome. J Thorac Cardiovasc Surg 1995;110:1125-9.

6. Massard G, Lyons G, Wihlm JM, Fernoux P, Dumont P, Kessler $\mathrm{R}$, et al. Early and long-term results after completion pneumonectomy. Ann Thorac Surg 1995;59:196-200.

7. Regnard JF, Icard $P$, Magdeleinat $P$, Jauffret $B$, Farés E, Levasseur P. Completion pneumonectomy: experience in eighty patients. J Thorac Cardiovasc Surg 1999;117:1095-101.

8. Martini N, Bains MS, Burt ME, Zakowski MF, McCormack P, Rusch VW, et al. Incidence of local recurrence and second primary tumors in resected stage I lung cancer. J Thorac Cardiovasc Surg 1995;109:120-9.

9. Deslauriers J. Indications for completion pneumonectomy. Ann Thorac Surg 1988;46:133

10. Snijder RJ, de la Riviére AB, Elbers HJJ, van den Bosch JMM. Survival in resected stage I lung cancer with residual tumor at the bronchial resection margin. Ann Thorac Surg 1998;65:212-6.

11. Riquet M, Manac'h D, Le Pimpec-Barthes F, Dujon A, Chehab A. Prognostic significance of surgical-pathologic N1 disease in nonsmall cell carcinoma of the lung. Ann Thorac Surg 1999;67:1572-6.

12. Yoshino I, Nakanishi R, Osaki T, Takenoyama M, Taga S, Hanagiri T, et al. Unfavorable prognosis of patients with stage II non-small cell lung cancer associated with macroscopic nodal metastases. Chest 1999;116:144-9.
13. Spaggiari L, Grunenwald DH, Girard P, Solli P, Chevalier TL. Pneumonectomy for lung metastases: indications, risks, and outcome. Ann Thorac Surg 1998;66:1930-3.

14. Agasthian T, Deschamps C, Trastek VF, Allen MS, Pairolero PC. Surgical management of bronchiectasis. Ann Thorac Surg 1996;62:976-80

15. Asamura H, Naruke T, Tsuchiya R, Goya T, Kondo H, Suemasu $\mathrm{K}$. What are the risk factors for arrhythmias after thoracic operations? A retrospective multivariate analysis of 267 consecutive thoracic operations. J Thorac Cardiovasc Surg 1993;106:110410.

16. Curtis JJ, Parker BM, McKenney CA, Wagner-Mann CC, Walls JT, Demmy TL, et al. Incidence and predictors of supraventricular dysrhythmias after pulmonary resection. Ann Thorac Surg 1998;66:1766-71.

17. Stamatis G, Eberhardt W, Stüben G, Bildat S, Dahler O, Hillejan L. Preoperative chemoradiotherapy and surgery for selected nonsmall cell lung cancer IIIB subgroups: long-term results. Ann Thorac Surg 1999;68:1144-9.

18. Cevese PG, Vecchioni R, D'Amico DF, Cordiano C, Biasiato R, Favia G, et al. Postoperative chylothorax. J Thorac Cardiovasc Surg 1975;69:966-71.

19. Sarsam MA, Rahman AN, Deiraniya AK. Postpneumonectomy chylothorax. Ann Thorac Surg 1994;57:689-90.

20. Wada H, Nakamura T, Nakamoto K, Maeda M, Watanabe Y. Thirty-day operative mortality for thoracotomy in lung cancer. J Thorac Cardiovasc Surg 1998;115:70-3. 